DrAft VERSION NOVEMBER 5, 2018

Preprint typeset using $\mathrm{L}^{A} \mathrm{~T}_{\mathrm{E}} \mathrm{X}$ style emulateapj v. 11/10/09

\title{
INTERNAL DYNAMICS AND MEMBERSHIP OF THE NGC 3603 YOUNG CLUSTER FROM MICROARCSECOND ASTROMETRY
}

\author{
Boyke Rochau $^{1}$, Wolfgang Brandner ${ }^{1}$, Andrea Stolte ${ }^{2}$, Mario Gennaro ${ }^{1}$, Dimitrios Gouliermis ${ }^{1}$, Nicola Da \\ Rio $^{1}$, Natalia Dzyurkevich ${ }^{1}$, and Thomas Henning ${ }^{1}$ \\ Draft version November 5, 2018
}

\begin{abstract}
We have analyzed two epochs of HST/WFPC2 observations of the young Galactic starburst cluster in NGC 3603 with the aim to study its internal dynamics and stellar population. Relative proper motions measured over 10.15 yrs of more than 800 stars enable us to distinguish cluster members from field stars. The best-fitting isochrone yields $A_{\mathrm{V}}=4.6-4.7 \mathrm{mag}$, a distance of $6.6-6.9 \mathrm{kpc}$, and an age of $1 \mathrm{Myr}$ for NGC 3603 Young Cluster (NYC). We identify pre-main-sequence/main-sequence transition stars located in the short-lived radiative-convective gap, which in the NYC occurs in the mass range $3.5-3.8 \mathrm{M}_{\odot}$. We also identify a sparse population of stars with an age of $4 \mathrm{Myr}$, which appear to be the lower mass counterparts to previously discovered blue supergiants located in the giant H II region NGC 3603. For the first time, we are able to measure the internal velocity dispersion of a starburst cluster from 234 stars with $I<18.5 \mathrm{mag}$ to $\sigma_{\mathrm{pm} 1 \mathrm{D}}=141 \pm 27 \mu \mathrm{as} \mathrm{yr}^{-1}\left(4.5 \pm 0.8 \mathrm{~km} \mathrm{~s}^{-1}\right.$ at a distance of $6.75 \mathrm{kpc}$ ). As stars with masses between 1.7 and $9 \mathrm{M}_{\odot}$ all exhibit the same velocity dispersion, the cluster stars have not yet reached equipartition of kinetic energy (i.e., the cluster is not in virial equilibrium). The results highlight the power of combining high-precision astrometry and photometry, and emphasize the role of NYC as a benchmark object for testing stellar evolution models and dynamical models for young clusters and as a template for extragalactic starburst clusters.

Subject headings: astrometry - open clusters and associations: individual (NGC 3603) - stars: evolution - stars: formation - stars: pre-main sequence - stars: kinematics and dynamics
\end{abstract}

\section{INTRODUCTION}

Massive young stellar clusters are outstanding objects containing copious numbers of stars over the entire stellar mass range. With masses between $10^{4} \mathrm{M}_{\odot}$ and $10^{7} \mathrm{M}_{\odot}$ (Zhang \& Fall 1999; de Grijs et al. 2003; Mengel et al. 2008), they cover the upper end of the cluster mass function and may constitute progenitors of globular clusters (GCs; Zhang \& Fall 1999; McCrady \& Graham 2007). While extragalactic starburst clusters, such as those in the Antennae Galaxies, are often barely resolved (Whitmore \& Schweizer 1995), in Milky Way starburst clusters thousands of individual stars can be observed.

In addition to three clusters in the Galactic Center region (Arches, Quintuplet, Young Nuclear Cluster), only a handful of Milky Way starburst clusters located in spiral arms have so far been identified (e.g. Brandner et al. 2008). Among the spiral arm clusters, the NGC 3603 Young Cluster (NYC), located in its namesake giant HiI region NGC 3603 (Kennicutt 1984), is the most compact and youngest cluster with an age of $\approx 1$ Myr (Brandl et al. 1999; Stolte et al. 2004; Sung \& Bessell 2004) and a central density $\rho_{0} \geq 6$. $10^{4} M_{\odot} \mathrm{pc}^{-3}$ (Harayama et al. 2008). It hosts three Wolf-Rayet stars, at least $6 \mathrm{O} 2 / \mathrm{O} 3$, and 30 late O-type stars (Moffat et al. 2004), and is extensively referenced as a template for extragalactic starburst environments (e.g. Lamers et al. 2006).

Previously, dynamical studies of Galactic starburst

\footnotetext{
${ }^{1}$ Max-Planck-Institute for Astronomy, Königstuhl 17, 69117 Heidelberg, Germany

${ }^{2}$ I. Physikalisches Institut, Universität zu Köln, Zülpicher Straße 77, 50937 Köln, Germany
}

clusters were largely restricted to one-dimensional velocity dispersions derived from radial velocity measurements of a few of the most luminous cluster members (e.g. Mengel \& Tacconi-Garman 2007). Using multiepoch observations of GCs, King \& Anderson (2001, 2002) and Anderson \& King (2003a) pioneered high precision proper motion studies with the Hubble Space Telescope $(H S T)$, which enabled the distinction of GC members from field stars, and the study of GC dynamics and kinematics.

In 2006, we initiated an extensive observational program to obtain multi-epoch high-angular resolution imaging observations of Galactic starburst clusters with the aim to study their internal dynamics and global motions. Here, we present the results of our analysis of two epochs of HST observations of NYC separated by $10.15 \mathrm{yr}$. Accurate proper motions enable us to get a "clean" census of the cluster population by rejecting field stars and to study the internal cluster dynamics.

\section{OBSERVATIONS AND DATA ANALYSIS}

Two epochs of observations of NGC 3603 with the HST/Wide-Field Planetary Camera 2 (WFPC2) separated by $10.15 \mathrm{yr}$ are analyzed. We compare Planetary Camera (PC1) observations in F547M and F814W from epoch 1 (GO 6763, archival data) with our second epoch observations in F555W and F814W (GO 11193). With an image scale of 45.5 mas pixel $^{-1}$, $\mathrm{PC} 1$ provides the best point-spread function (PSF) sampling of the WFPC2 cameras. While the first epoch observations were carried out in stare mode, for the second epoch we selected a four-point sub-pixel dither pattern to facilitate bad pixel recovery and to provide an improved PSF sampling. Table 1 summarizes the observations, including individual 

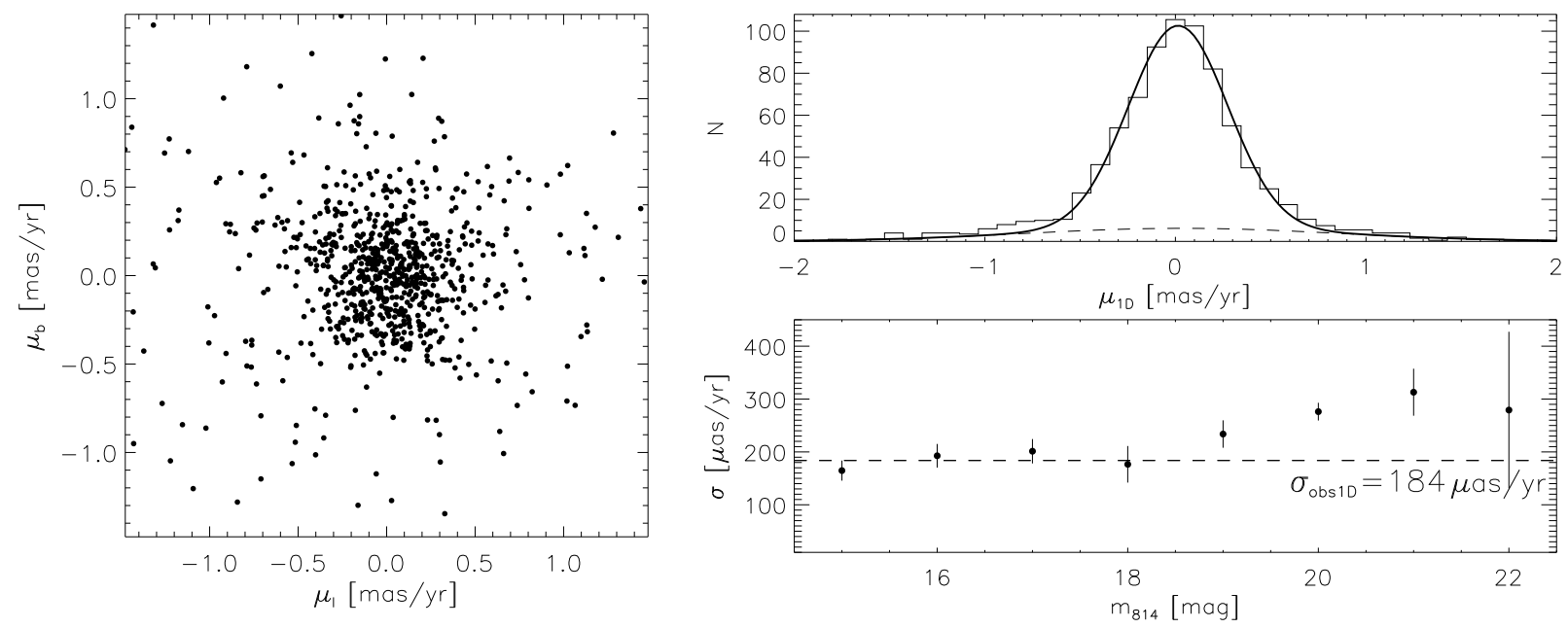

FIG. 1. - Left: proper motions of stars in the NYC reference frame in Galactic coordinates. Right: histogram of the averaged onedimensional proper motions of all stars (top), fitted by a two-component Gaussian (thick line), while the dashed line represents the distribution of the field stars. The lower panel depicts the observed proper motion dispersion as a function of stellar magnitude of the cluster member candidates. For $14.5 \mathrm{mag}<\mathrm{m}_{814}<18.5 \mathrm{mag}$, we obtain a standard deviation $\sigma_{\mathrm{obs} 1 \mathrm{D}}=184 \mu$ as $/ \mathrm{yr}$ with an uncertainty of $20 \mu \mathrm{as} / \mathrm{yr}$ (dashed line).

TABLE 1

OBSERVING LOG

\begin{tabular}{ccccc}
\hline Date & Filter & $t_{\text {exp }}(\mathrm{s})$ & $\mathrm{t}_{\text {tot }}(\mathrm{s})$ & Stars \\
\hline $30 / 07 / 97$ & F547M & $3 \times 1,12 \times 10,8 \times 30$ & 363 & 772 \\
$31 / 07 / 97$ & F814W & $3 \times 0.4,12 \times 5,8 \times 20$ & 221 & 1163 \\
$26 / 09 / 07$ & F555W & $4 \times 0.4,4 \times 26,4 \times 100$ & 506 & 1048 \\
$26 / 09 / 07$ & F814W & $4 \times 18,4 \times 160$ & 712 & 2014 \\
\hline \hline
\end{tabular}

and total exposure times.

Data reduction has been performed with IRAF/Pyraf. We combined the individual bias subtracted, flatfielded images with identical exposure times using multidrizzle (Koekemoer et al. 2002). This corrects for velocity aberration and geometrical distortion including the 34th column anomaly based on the latest distortion correction ${ }^{3} 4$. For the second epoch observations we applied $2 \times 2$ oversampling.

Astrometry and photometry were derived from the drizzled images for each filter and exposure time setting using DAOPHOT (Stetson 1990) with a Penny2 PSF varying linearly across the field. Near the faint end, where the photometric uncertainties start to increase, the star list was filled in by the results derived from the next longer exposure, and uncertainties assessed accordingly. The final number of detections in each band and epoch for a $5 \sigma$ threshold above the background noise is listed in Table 11. Photometric calibration is based on the zero points provided by Dolphin (2000). Photometric correction for charge transfer efficiency follows the recipe provided by A. Dolphin 5 , and the astrometric correction is based on Equation 7 of Kozhurina-Platais et al. (2007) with the values for $b_{1}, b_{2}$, and $b_{3}$ given in Table 2 (note the different pixel scale due to $2 \times 2$ oversampling for the second epoch).

The combined image has distortions reduced to a level of 0.02 pixel (see Anderson \& King 2003b). Due

\footnotetext{
3 http://www.stsci.edu/hst/wfpc2/analysis/calfiles

4 http://ftp.stsci.edu/cdbs/uref/sad1946fu_idc.fits

5 http://purcell.as.arizona.edu/wfpc2_calib/
}

to the different orientation angle of $51^{\circ}$ between the two epochs, we have to consider the uncertainty of the position induced by the residual geometric distortion, $\sigma_{\text {geo }}=0.017 \pm 0.001$ pixel. For sinusoidal pixel phase errors (see Anderson \& King 2000, their Figure 2), the second epoch dithering pattern with 0.5 pixel shifts largely cancels out the pixel phase error. As the first epoch was observed in stare mode, the pixel phase error has to be considered. With a typical amplitude of the sinusoidal pixel phase error of \pm 0.02 pixel, the uncertainty to be included in our analysis amounts to an average residual uncertainty of $\sigma_{\text {pxph }}=0.013 \pm 0.003$ pixel. Simulations based on TinyTim PSFs (Krist 1995) indicate that the positional PSF fitting uncertainty results in a centroiding error of $\sigma_{\mathrm{PSF}}=0.013 \pm 0.001$ pixel. The effect of $H S T$ breathing on pixel scale was determined by measuring the separation of wide pairs of stars on frames obtained during different phases of HST's orbit, resulting in $\sigma_{\text {breath }}=0.009 \pm 0.002$ pixel. The combined contribution of these effects amounts to $\sigma_{\text {err }}=$ $\sqrt{\sigma_{\text {geo }}^{2}+\sigma_{\text {pxph }}^{2}+\sigma_{\mathrm{PSF}}^{2}+\sigma_{\text {breath }}^{2}}=1.21 \pm 0.18$ mas. The observed proper motion dispersion has to be corrected for $\sigma_{\text {err }}$ to derive the intrinsic velocity dispersion of the cluster members.

TABLE 2

PARAmeters of the ASTROMETRIC CORRECTION

\begin{tabular}{cccc}
\hline Epoch & $b_{1}(\mathrm{px})$ & $b_{2}\left(\mathrm{px} \mathrm{mag}^{-1}\right)$ & $b_{3}$ \\
\hline 1997.58 & 0.094 & -0.0056 & $6.17 \times 10^{-5}$ \\
2007.73 & 0.122 & -0.011 & $3.31 \times 10^{-5}$ \\
\hline \hline
\end{tabular}

As the orientation angles of the two epochs differ $\left(\Delta \Theta=51^{\circ}\right)$, the common field available to our analysis is a circle with a diameter of $30^{\prime \prime}$. A geometric transformation based on a preliminary list of main-sequence (MS) cluster members is derived using IRAF / GEOMAP with a second-order polynomial. Stars detected in $I$ band with photometric PSF fitting uncertainties $\sigma_{\text {phot }}<0.1$ mag are matched after applying this transformation, and in- 
dividual proper motions are calculated for each star. In this reference frame, cluster members center around $(0,0)$ in the proper motion vector point diagram. The final proper motion table contains 829 matched stars.

\section{PROPER MOTIONS AND MEMBERSHIP}

In Figure 1 (left) we show the measured proper motions for all stars with respect to the cluster reference frame in Galactic coordinates. The symmetrical distribution of all proper motions around $(0,0)$ indicates the absence of any large relative motion of the cluster with respect to the field, i.e., the cluster follows the Galactic rotation curve. As NYC's Galactic longitude of $l=291.6^{\circ}$ implies an almost tangential view into the Carina spiral arm, the distributions of cluster member and field star proper motions are superimposed. This proper motion distribution is fitted by a two-component Gaussian with the wide function describing predominantly foreground stars, and hence non-cluster members (dashed line in Figure 1, top right), and the narrow component is interpreted as cluster member candidates. We calculate cluster membership probabilities $P_{\text {mem }}$ as described in Jones \& Walker (1988) and consider stars as cluster members if $P_{\text {mem }}>0.9$. This significantly reduces the number of contaminating field stars, but due to the similar proper motions of cluster and field, some field stars might remain in the cluster sample. Based on Besançon models (Robin et al. 2003), we estimate the number of field stars in our field of view to be 46 stars between $16 \mathrm{mag}<\mathrm{m}_{555}<25 \mathrm{mag}$. Including stars with a membership probability above 0.9 in our cluster sample, we subtracted a total of 58 stars as field stars. For the variable stars $H S T 12,474,481$, and 574, studied by Moffat et al. (2004), we found membership probabilities $P_{\text {mem }}$ of $0.75,0.98,0.90$, and 0.98 , respectively, indicating that the latter three are likely cluster members.

\section{EXTINCTION, DISTANCE AND AGE}

The $m_{555}, m_{555}-m_{814} \mathrm{CMD}$ is shown in Figure 2 (left). The distinction between candidate cluster members (small dots) and field stars (open circles) as described in Section 3 leads to a very well defined cluster sequence with high-mass MS stars, intermediate-mass stars located in the pre-main-sequence (PMS)-MS transition region between $m_{555}=18 \mathrm{mag}$ and $20 \mathrm{mag}$, and more than 300 lower mass PMS stars down to $m_{555}=24.5 \mathrm{mag}$ (Figure 2, middle). The efficiency of the proper motion member selection is evident in the left panel of Figure 2, particularly among PMS and faint stars that could not be distinguished from cluster members from their colors alone. The foreground sequence blueward of the PMS does likely not belong to the cluster, suggesting a residual contamination of 18 stars with $20 \mathrm{mag}<m_{555}<24 \mathrm{mag}$.

In the following, we assume solar metallicity (Melena et al. 2008) for the cluster and the relation between absolute and selective extinction from Schlegel et al. (1998). The upper MS is well fitted by a 1 Myr Padova isochrone (Marigo et al. 2008, black solid line along the MS in the right panel of Figure 2) for $A_{\mathrm{V}}=4.7 \mathrm{mag}$ and a distance modulus of $14.1 \mathrm{mag}$. For the analysis of the PMS-MS transition region and the lower mass PMS we use Siess models (Siess et al. 2000), transformed by Da Rio et al. (2009, S00/DR), as well as PISA-FRANEC models (Degl'Innocenti et al. 2008, PF09), transformed into the observational plane using ATLAS 9 model atmospheres (Castelli \& Kurucz 2003). The best-fitting isochrones yield a distance between $6.6 \mathrm{kpc}$ (PF09) and $6.9 \mathrm{kpc}(\mathrm{S} 00 / \mathrm{DR})$ and a visual extinction $A_{\mathrm{V}}=4.7 \mathrm{mag}$ (PF09) and $4.6 \mathrm{mag}$ (S00/DR), respectively for an age of $1 \mathrm{Myr}$. We note that the derived selective extinction is in good agreement with $E(\mathrm{~B}-\mathrm{V})=1.25 \mathrm{mag}$ as reported by Sung \& Bessell (2004), though the absolute extinction value derived by us is slightly higher due to the use of the Schlegel et al. relations. The $1 \mathrm{Myr}$ PF09 isochrone represents the PMS best, in particular at the PMS-MS transition region.

At an age of $1 \mathrm{Myr}$, stars with masses between 3.5 and $3.8 \mathrm{M}_{\odot}$ are expected to be in the short-lived radiativeconvective $(\mathrm{r}-\mathrm{c})$ gap phase (Mayne et al. 2007). This phase corresponds to the formation of a radiative core in the interior of the stars, due to the increasing central temperature (Iben 1965). We observe eight sources in the $\mathrm{r}-\mathrm{c}$ gap at $18.5 \mathrm{mag}<m_{555}<19 \mathrm{mag}$ (Figure 2) and $m_{555}-m_{814} \sim 2.25 \mathrm{mag}$ (shown as diamonds in the right panel of Figure 2). If their PMS nature is spectroscopically confirmed, this is the first identification of PMS stars in this interesting evolutionary stage.

A previously unreported CMD feature is the apparent extension of the MS toward lower masses below the PMS-MS transition region $\left(m_{555} \gtrsim 18.5 \mathrm{mag}\right)$. Isochrone fitting to the MS turn-on yields an age of $4 \mathrm{Myr}$. The derived age is consistent with recent estimates of the age of the two blue supergiants Sher 23 and Sher 25 (Melena et al. 2008). These stars might represent an earlier epoch of star formation in the giant H II region (see also Sung \& Bessell 2004).

\section{VELOCITY DISPERSION AND CLUSTER DYNAMICS}

The distribution $\sigma_{\mathrm{obs} 1 \mathrm{D}}$ of the proper motions $\mu_{\mathrm{obs} 1 \mathrm{D}}=$ $\frac{\mu_{l}+\mu_{b}}{2}$, as shown in the upper right panel of Figure 1, is a combination of internal velocity dispersion and instrumental effects, resulting in $\sigma_{\mathrm{obs} 1 \mathrm{D}}=\sqrt{\sigma_{\mathrm{pm} 1 \mathrm{D}}^{2}+\sigma_{\mathrm{err}}^{2}}$. In the lower right panel of Figure 1 we show the observed one-dimensional proper motion dispersion as a function of stellar magnitude in bins of 1 mag for candidate cluster members. The velocity dispersion is constant for stars with $14.5 \mathrm{mag} \leq \mathrm{m}_{\mathrm{F} 814 \mathrm{~W}} \leq 18.5 \mathrm{mag}\left(\approx 1.7-9 \mathrm{M}_{\odot}\right)$.

Correcting the observed one-dimensional proper motion dispersion of $\sigma_{\text {obs1D }}=184 \pm 20 \mu \mathrm{as} / \mathrm{yr}$ for the instrumental effects discussed in Section 2 results in an intrinsic one-dimensional velocity dispersion $\sigma_{\mathrm{pm} 1 \mathrm{D}}=141 \pm 27 \mu \mathrm{as}$ $\mathrm{yr}^{-1}$ for stars brighter than $m_{814} \approx 18.5 \mathrm{mag}$, assuming a negligible effect of binary orbital motions (Girard et al. 1989). This corresponds to $\sigma_{\text {cl1D }}=4.5 \pm 0.8 \mathrm{~km} \mathrm{~s}^{-1}$ at a distance of $6.75 \mathrm{kpc}$. The constant velocity dispersion for stars in the mass range $1.7-9 \mathrm{M}_{\odot}$ indicates a lack of equipartition of energy among cluster members. This provides a strong indication that NYC is far from virial equilibrium.

Nevertheless, an upper limit of the cluster mass can be obtained by deriving the virial mass $M_{d y n}$ from the observed velocity dispersion (Spitzer 1987):

$$
\mathrm{M}_{\mathrm{dyn}}=\eta \frac{r_{\mathrm{h}} \sigma_{\mathrm{cl} 3 \mathrm{D}}^{2}}{\mathrm{G}}
$$

where $\eta \approx 2.5$ (weakly depending on cluster density 


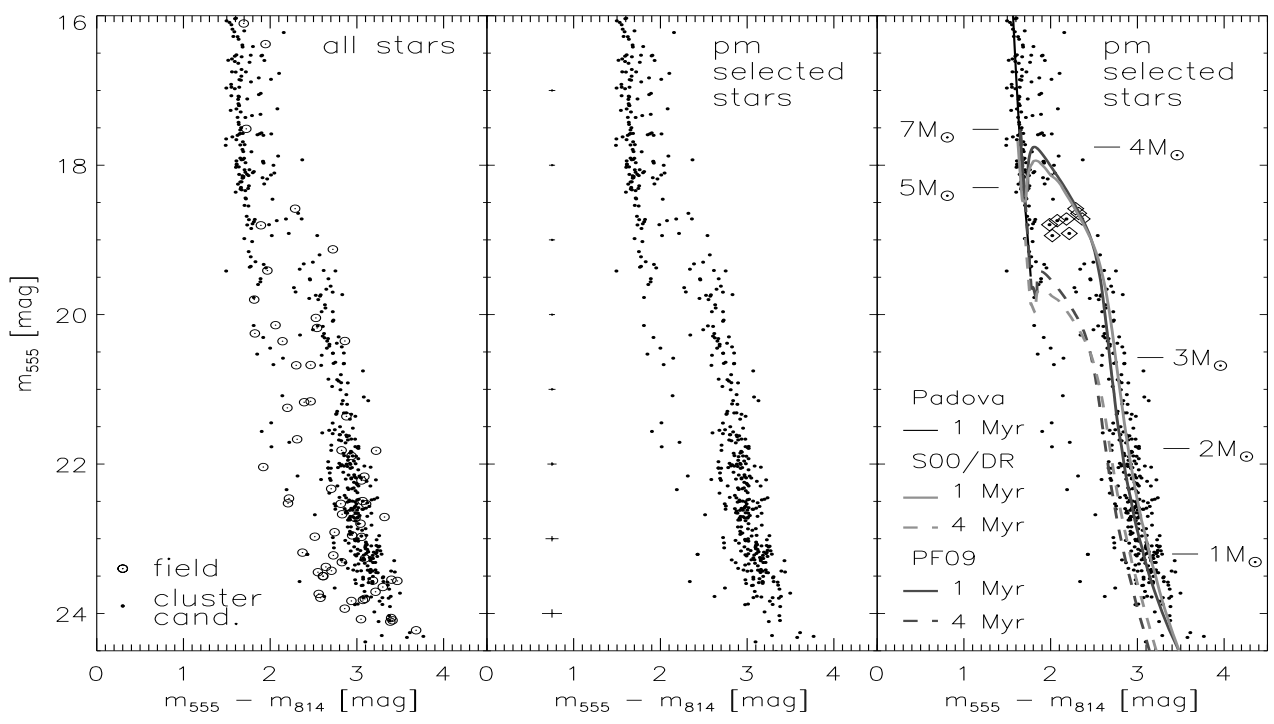

FIG. 2. - Left: $m_{555}-m_{814}$ vs. $m_{555}$ CMD of cluster (small dots) and field stars (open circles). Clearly visible are the MS and PMS loci at $m_{555}-m_{814} \sim 1.5$ and $3 \mathrm{mag}$, respectively. Center: cluster member candidates with $P_{\mathrm{mem}}>0.9$. Apparent is the narrow PMS after the proper motion selection. Right: cluster member candidates with the best-fitting Padova 1 Myr (MS, black solid line) and PF09 1 Myr as well as S00/DR 1 Myr (PMS, dark and light gray solid lines, respectively) isochrones overplotted. Diamonds mark the stars in the r-c gap. The extension of the MS below the transition region is not covered by the younger isochrones, but is reproduced by a 4 Myr isochrone (PF09, dark gray dashed line; S00/DR, light gray dashed line).

structure), $r_{\mathrm{h}}$ is the half-mass radius, $\sigma_{\mathrm{cl} 3 \mathrm{D}}$ is the threedimensional velocity dispersion, and $G$ is the gravitational constant.

NYC is mass segregated with its core radius increasing with decreasing stellar mass (Nürnberger \& Petr-Gotzens 2002). A lower limit on $r_{\mathrm{h}}$ for the high-mass stars as derived from $H S T$ data is comparable to the core radius of $\approx 0.2 \mathrm{pc}$ (Stolte 2003), whereas Harayama et al. (2008) based on the analysis of near-infrared adaptive optics data estimate $r_{\mathrm{h}}=$ $0.7-1.5 \mathrm{pc}$ for stars in the mass range $0.5-2.5 \mathrm{M}_{\odot}$. If we assume $r_{\mathrm{h}}=0.5 \mathrm{pc}$ and a three-dimensional velocity dispersion of $\sigma_{\mathrm{cl} 3 \mathrm{D}}=\sqrt{3} \times 4.5 \pm 0.8 \mathrm{~km} \mathrm{~s}^{-1}$, we derive $M_{\text {dyn }}=17600 \pm 3800 \mathrm{M}_{\odot}$.

Considering that this dynamical mass estimate provides an upper limit, it is in agreement with photometric studies of NYC, which assigned masses to individual stars, and estimated the total stellar mass to $M_{c l} \approx 10000-16000 \mathrm{M}_{\odot} \quad$ (Stolte et al. 2006; Harayama et al. 2008).

\section{SUMMARY AND CONCLUSIONS}

Based on two epochs of high-accuracy astrometric $H S T$ /WFPC2 observations separated by $10.15 \mathrm{yr}$, relative proper motions of 829 stars were measured. A selection of candidate cluster members with $P_{\text {mem }}>0.9$ results in a clean cluster CMD. The best-fitting isochrone yields an age of $1 \mathrm{Myr}$, a distance of $6.6-6.9 \mathrm{kpc}$, and $A_{\mathrm{V}}=4.6-4.7 \mathrm{mag}$ for the PMS and intermediate-mass MS cluster members.

Stars at the location of the short-lived radiative convective gap, which occurs at $3.5-3.8 \mathrm{M}_{\odot}$ at the age of NYC, are identified for the first time. We find hints of a sparse young low-mass population with an age of $\sim 4$ Myr, which might constitute an earlier generation of star formation in NGC 3603, and likely represents the low-mass counterparts to several blue supergiants in the vicinity of NYC.

For the first time, the internal velocity dispersion of the starburst cluster NYC could be measured. For stars with masses $1.7 \mathrm{M}_{\odot}<\mathrm{M}<9 \mathrm{M}_{\odot}$, we determine a onedimensional velocity dispersion of $141 \pm 27 \mu \mathrm{as} \mathrm{yr}^{-1}$, corresponding to $4.5 \pm 0.8 \mathrm{~km} \mathrm{~s}^{-1}$ at a distance of $6.75 \mathrm{kpc}$. From the fact that the velocity dispersion does not vary with stellar mass in this mass range, we deduce that NYC has not yet reached equipartition of energy. This is not entirely unexpected at the young age of the cluster, since its crossing time is estimated to be $1.4 \mathrm{Myr}$ by Nürnberger \& Petr-Gotzens (2002).

The same might be true for many extragalactic starburst clusters, where mass estimates rely on the measurements of velocity dispersions. If these clusters are also not yet in virial equilibrium, their masses might be systematically overestimated. Thus, NYC provides an important benchmark for our understanding of the early dynamical evolution and the long-term survival of young, massive stellar clusters in the Milky Way and in other galaxies.

We thank Will Clarkson for many useful comments and discussions. We further thank the referee whose comments and suggestions helped in improving this Letter. We acknowledge support from the Deutsches Zentrum für Luft- und Raumfahrt (DLR), Förderkennzeichen 50 OR 0401. A.-S. and D.-G. acknowledge the support of the German Research Foundation (DFG) through the Emmy Noether grant STO 496/3-1 and the grant GO $1659 / 1-2$, respectively.

\section{REFERENCES}


Brandl, B., Brandner, W., Eisenhauer, F., Moffat, A. F. J., Palla, F., \& Zinnecker, H. 1999, A\&A, 352, L69

Brandner, W., Clark, J. S., Stolte, A., Waters, R., Negueruela, I. \& Goodwin, S. P. 2008, A\&A, 478, 137

Castelli, F., \& Kurucz, R. L. 2003, in IAU Symp. 210, Modelling of Stellar Atmospheres, ed. N. Piskunov, W. W. Weiss, \& D. F. Gray (Cambridge: Cambridge Univ. Press), 20P

Da Rio, N., Gouliermis, D. A., \& Henning, T. 2009, ApJ, 696, 528

Degl'Innocenti, S. \& Prada Moroni, P. G. \& Marconi, M. \& Ruoppo, A. 2008, Ap\&SS, 316, 25

de Grijs, R., Anders, P., Bastian, N., Lynds, R., Lamers,

H. J. G. L. M., \& O'Neil, E. J. 2003, MNRAS, 343, 1285

Dolphin A. E., 2000, PASP, 112, 1383

Girard, T. M., Grundy, W. M., Lopez, C. E., \& van Altena, W. F. 1989, AJ, 98, 227

Harayama, Y., Eisenhauer, F., \& Martins, F. 2008, ApJ, 675, 1319

Iben, I., Jr. 1965, ApJ, 141, 993

Jones, B. F., \& Walker, M. F. 1988, AJ, 95, 1755

Kennicutt, R. C., Jr. 1984, ApJ, 287, 116

King, I. R., \& Anderson, J. 2002, A\&A, 273, 167

King, I. R., \& Anderson, J. 2001, A\&A, 228, 19

Koekemoer, A. ., Fruchter, A. S., Hook, R. N., \& Hack, W. 2002 The 2002 HST Calibration Workshop, 337

Kozhurina-Platais, V., Goudfrooij, \& Puzia, T. H. 2007, Instrument Science Report ACS 2007-04

Krist, J. 1995, in ASP Conf. Ser. 77, Astronomical Data Analysis Software and Systems IV., ed. R. A. Shaw, H. E. Payne, \& J. J. E. Hayes (San Francisco, CA: ASP), 349

Lamers, H. J. G. L. M., Anders, P., \& de Grijs, R. 2006, A\&A, 452,131
Marigo, P., Girardi, L., Bressan, A., Groenewegen, M. A. T., Silva, L. \& Granato, G. L. 2008, A\&A, 482, 883

Mayne, N. J., Naylor, T., Littlefair, S. P., Saunders, E. S., \& Jeffries, R. D 2007, MNRAS, 375, 1220

McCrady, N., \& Graham, J. R. 2007, ApJ, 663, 844

Melena, N. W., Massey, P., Morrell, N. I., \& Zangari, A. M. 2008, AJ, 135,878

Mengel, S., Lehnert, M. D., Thatte, N. ., Vacca, W. D.,

Whitmore, B., \& Chandar, R. 2008, A\&A, 489, 1091

Mengel, S., \& Tacconi-Garman, L. E. 2007, A\&A, 466, 151

Moffat, A. F. J., Poitras, V., Marchenko, S. V., Shara, M. M., Zurek, D. R., Bergeron, E., \& Antokhina, E. A. 2004, AJ, 128, 2854

Nürnberger, D. E. A., \& Petr-Gotzens, M. G. 2002, A\&A, 382537

Robin, A. C., Reylé, C., Derrière, S., \& Picaud, S. 2003, A\&A, 409,523

Schlegel, D. J., Finkbeiner, D. P., \& Davis, M. 1998, ApJ, 500, 525

Siess, L., Dufour, E., \& Forestini, M. 2000, A\&A, 358, 593

Spitzer, L. 1987, Dynamical evolution of globular clusters (Princeton, NJ: Princeton Univ. Press), 11P

Stetson, P. B. 1990, PASP, 102, 932

Stolte, A. 2003, Mass function and Mass Segregation in Young Starburst Clusters, PhD thesis (University of Heidelberg)

Stolte, A., Brandner, W., Brandl, B., Zinnecker, H., \& Grebel, E. K. 2004, AJ, 128, 765

Stolte, A., Brandner, W., Brandl, B., \& Zinnecker, H. 2006, AJ, 132,253

Sung, H., \& Bessell, M. S. 2004, AJ, 127, 1014

Whitmore, B. C., \& Schweizer, H. W. 1995, AJ, 109, 960

Zhang, Q., \& Fall, S. M. 1999, ApJ, 527, L81 